\begin{tabular}{|c|l|}
\hline Title & $\begin{array}{l}\text { Microscopic coexistence of superconductivity and charge order in the organic superconductor beta"-(BEDT- } \\
\text { TTF)(4)[(H30)Ga(C20 4)(3)]center dot C6H5NO2 }\end{array}$ \\
\hline Author(s) & Ihara, Y.; Moribe, K.; Fukuoka, S.; Kawamoto, A. \\
\hline Citation & $\begin{array}{l}\text { Physical Review B, 100(6), 060505 } \\
\text { https://doi.org/10.1103/PhysRevB.100.060505 }\end{array}$ \\
\hline Issue Date & 2019-08-20 \\
\hline Doc URL & http://hdl.handle.net/2115/75730 \\
\hline Rights & O2019A A merican Physical Society \\
\hline Type & article \\
\hline File Information & PhysRevB.100.060505.pdf \\
\hline
\end{tabular}

Instructions for use 


\title{
Microscopic coexistence of superconductivity and charge order in the organic superconductor $\beta^{\prime \prime}-(\mathrm{BEDT}-\mathrm{TTF})_{4}\left[\left(\mathrm{H}_{3} \mathrm{O}\right) \mathrm{Ga}\left(\mathrm{C}_{2} \mathrm{O}_{4}\right)_{3}\right] \cdot \mathrm{C}_{6} \mathrm{H}_{5} \mathrm{NO}_{2}$
}

\author{
Y. Ihara, ${ }^{*}$ K. Moribe, S. Fukuoka, and A. Kawamoto \\ Department of Physics, Graduate School of Science, Hokkaido University, Sapporo 060-0810, Japan
}

(Received 24 January 2019; revised manuscript received 19 May 2019; published 20 August 2019)

\begin{abstract}
An electron paramagnetic resonance study for an organic superconductor $\beta^{\prime \prime}$-(BEDT-TTF $)_{4}$ $\left[\left(\mathrm{H}_{3} \mathrm{O}\right) \mathrm{Ga}\left(\mathrm{C}_{2} \mathrm{O}_{4}\right)_{3}\right] \cdot \mathrm{C}_{6} \mathrm{H}_{5} \mathrm{NO}_{2}$ reveals that superconductivity coexists uniformly with the charge ordered state in one material. In the charge ordered state, the interplane spin exchange is gapped, while the in-plane conductivity is not significantly modified. This anisotropic behavior is explained by an exotic charge ordered state, in which molecular-site-selective carrier localization coexists with conducting carriers on other molecules. The relationship between superconductivity and this conductive charge ordered state is investigated.
\end{abstract}

DOI: 10.1103/PhysRevB.100.060505

The metal-insulator transition in a partially filled conduction band is caused by strong electron-electron interactions. In the case of a half-filled band, strong on-site Coulomb repulsion prevents the carriers from moving to stabilize the MottHubbard insulating state, while in a quarter-filled band, the long-range Coulomb interaction is responsible for the charge ordered (CO) insulating state. Superconductivity sometimes appears in the vicinity of the CO state [1-3], leading us to address that long-range electron-electron interactions can be the source of the superconducting (SC) pairing interaction in the same manner as the on-site interaction contributes to form unconventional superconductivity near the magnetic instability [4]. Since many unconventional superconductors have been found near the magnetic critical points, we naturally expect that intriguing SC features would be found for superconductivity near the charge instability. However, an experimental realization is difficult because the charge ordering coincides with strong carrier localization, which severely conflicts with the itinerant nature of the SC state. Nonetheless, a theoretical study based on the extended Hubbard model shows that charge fluctuations near the charge ordering transition can contribute to the SC pair formation when the charge ordering transition is suppressed to very low temperatures [5]. In fact, an increase in charge fluctuations is observed in the organic superconductor from an optical study [6], suggesting the interplay between the charge instability and superconductivity. Further experimental identification for the relationship between the neighboring, and even coexisting $\mathrm{CO}$ and $\mathrm{SC}$ states is crucial to expand the possibility of a SC pairing mechanism.

To investigate experimentally the effect of long-range electron-electron interactions on superconductivity, layered organic superconductors are the best suited because of their low carrier density, and thus a weak screening effect. Several organic conductors, such as $\alpha$-type and $\theta$-type BEDT-TTF salts (BEDT-TTF: bis(ethylenedithio)tetrathiafulvalene) show apparent charge ordering transitions [3,7-9]. Theoretical studies for $\theta$-type salts proposed a threefold CO state [10], which

\footnotetext{
*yihara@phys.sci.hokudai.ac.jp
}

can maintain metallic conductivity even when some parts of the carriers are localized at one of three molecular sites. Further theoretical studies show that when itinerant carriers interact with localized charges, an unconventional metallic state referred to as a pinball liquid state will be realized [11-13]. Experimentally, however, such a threefold CO state has not been found in $\theta$-type salts [14], because the threefold $\mathrm{CO}$ state appears only in a limited parameter space, where the stability of the horizontal stripe phase competes with the vertical phase $[10,11]$. To reveal the novel CO state and its impact on SC pair formation, we should study materials which show a nontrivial $\mathrm{CO}$ state very close to the SC transition temperature.

A layered organic superconductor $\beta^{\prime \prime}$ (BEDT-TTF $)_{4}\left[\left(\mathrm{H}_{3} \mathrm{O}\right) \mathrm{Ga}\left(\mathrm{C}_{2} \mathrm{O}_{4}\right)_{3}\right] \cdot \mathrm{C}_{6} \mathrm{H}_{5} \mathrm{NO}_{2} \quad\left(\beta^{\prime \prime}-\mathrm{Ga}\right)$ salt [15] is an ideal material to explore charge order and superconductivity, because this compound shows charge instability at a temperature very close to the SC transition temperature $T_{c}=7 \mathrm{~K}$. Previous NMR and electron paramagnetic resonance (EPR) experiments detected no anomaly due to a magnetic phase transition at the charge ordering temperature $T_{\mathrm{CO}}=8.5 \mathrm{~K} \quad[16,17]$, which was determined as the onset of NMR spectrum splitting [18]. Also, the NMR experiments showed that low-energy spin dynamics increases at $T_{\mathrm{CO}}$, which is suggestive of an increase in the charge fluctuations near the SC transition [16]. Besides, from the NMR intensity ratio between the charge-rich and charge-poor sites, a threefold charge pattern has been suggested [18]. As charge localization in the CO state coexists with metallic conductivity, and even with superconductivity below $T_{c}, \beta^{\prime \prime}-\mathrm{Ga}$ salt is one of the best candidates for the experimental realization of the pinball liquid state. However, as the resistivity experiments suggest the possibility of phase segregation, that is, the $\mathrm{CO}$ part of the sample is separated from the SC part [19], we should clarify if the CO state coexists or does not coexist microscopically with superconductivity. NMR spectroscopy is one of the most powerful techniques to investigate the electronic state from a microscopic viewpoint. However, because of an insufficient spectrum resolution, we were not able to exclude 

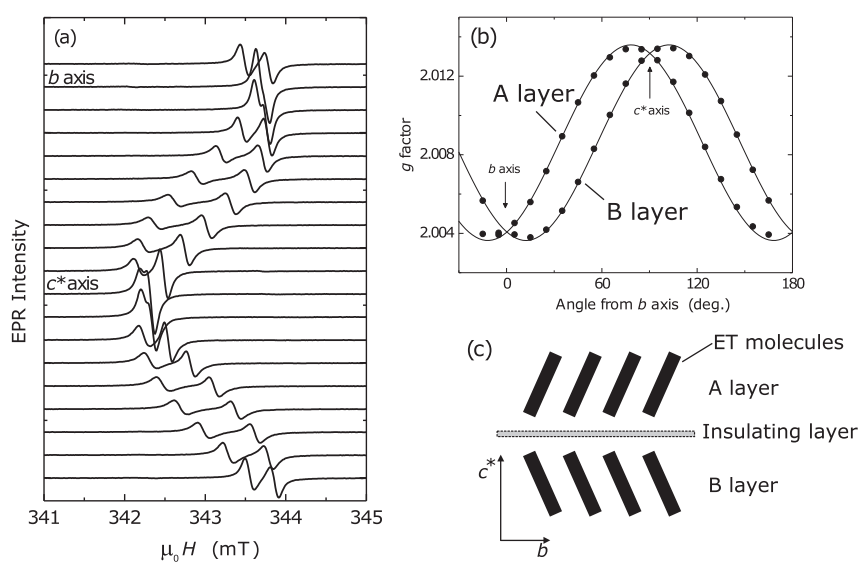

FIG. 1. (a) $X$-band EPR spectra of $\beta^{\prime \prime}$-Ga salt at $3.6 \mathrm{~K}$ in various external field directions. The two-peak spectrum observed in the arbitrary field direction merges into a single peak in fields along the symmetric axes. (b) Field direction dependence of the $g$ factors determined from the EPR peak positions. Two branches with a sinusoidal angle dependence originate from the A and B layers. (c) The double-layer crystal structure of $\beta^{\prime \prime}$-Ga salt. The conducting BEDT-TTF molecule layers are separated by the insulating anion layers. The long axis of the BEDT-TTF molecules in layer A (B) is inclined by $76^{\circ}\left(-76^{\circ}\right)$ with respect to the $b$ axis, which coincides with the maximum in the angle dependence of the $g$ factor (b).

the possibility of phase segregation. An alternative probe with higher resolution was desired.

In this Rapid Communication, we show that the $X$-band EPR experiment is one such probe. The EPR signal in $\beta^{\prime \prime}-\mathrm{Ga}$ salt originates from the $\pi$ electrons in the highest occupied molecular orbital of the BEDT-TTF molecules. We succeed in detecting the charge anomaly on the clearly resolved EPR spectrum by taking advantage of the anisotropy of $g$ factors and the bilayer crystal structure of $\beta^{\prime \prime}$-Ga salt. Thus, the EPR experiment allows us to observe the phase segregation, if any, as an additional component of the EPR spectrum. The present results, which are explained by a single EPR contribution at any temperature, clearly evidence a uniform coexistence between the $\mathrm{SC}$ and $\mathrm{CO}$ states. We also conducted a resistivity measurement to confirm that the $\mathrm{CO}$ state actually involves the conducting carriers, because the EPR experiment is in principle a spin-sensitive probe. The results of resistivity and EPR measurements are explained consistently, which unambiguously suggest a SC state coexisting with the conductive CO state.

Single-crystalline samples were grown by the standard electrochemical reaction [15]. The $X$-band EPR experiments were performed with a commercial spectrometer (Bruker EMX Plus). One single crystal with a dimension of $2 \times 0.5 \times$ $0.2 \mathrm{~mm}^{3}$ was used for the experiment. The orientation of the external magnetic field was tuned by a single axis rotator, with which the sample can be rotated in situ around the crystalline $a$ axis. We also performed in-plane ( $b$ axis) and interplane ( $c^{*}$ axis) resistivity measurements by the conventional fourprobe method [20].

Figure 1(a) shows the EPR spectra at the lowest temperature of $3.6 \mathrm{~K}$ in the $b c^{*}$ plane fields. The field direction is determined by the angle from the $b$ axis. The angular

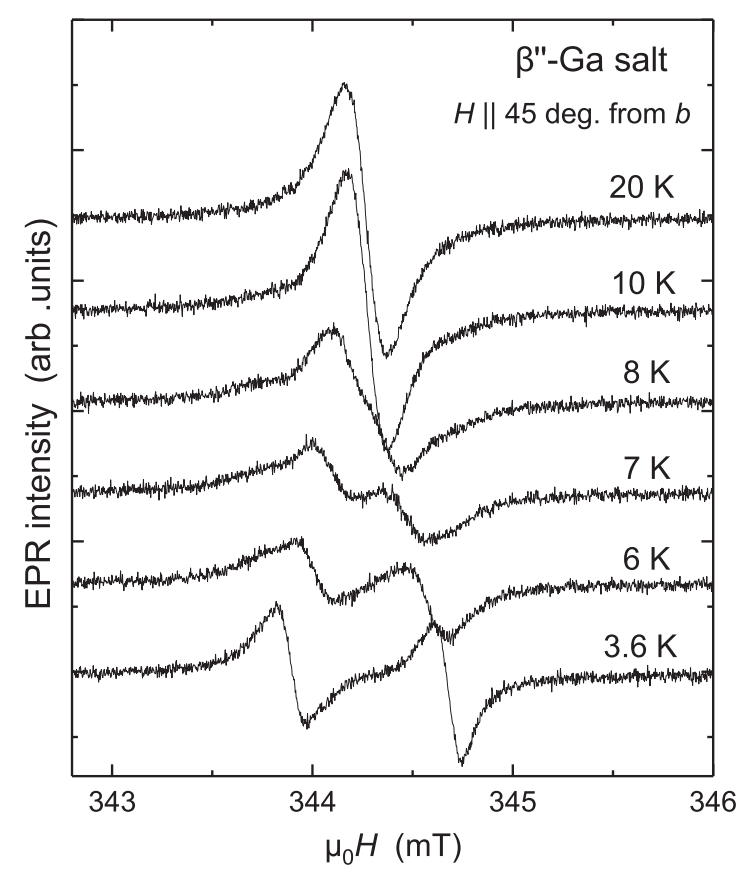

FIG. 2. The EPR peak splitting observed below $T_{\mathrm{CO}}=8.5 \mathrm{~K}$ in the $45^{\circ}$ field, where the peak separation at the lowest temperature becomes the maximum. The spectrum splitting without the increase in the spectrum width indicates an anomaly in the interplane cross relaxation (see text) with a negligible modification in the in-plane spin relaxation.

dependence of the EPR spectrum originates from the anisotropic $g$ tensor, for which the principal axes coincide with the symmetric axes of the BEDT-TTF molecule, and the principal values were determined for $\beta$-(BEDT-TTF $)_{2} \mathrm{I}_{3}$ salt as $g=(2.011,2.008,2.002)$ [21]. The $g$ factors for each field direction were determined by fitting the spectra with a two-peak Lorenzian function. As the result, two sinusoidal branches were obtained as shown in Fig. 1(b). These branches are assigned to the EPR signals from the A and B layers displayed in Fig. 1(c). Assuming that the principal axes of the $g$ tensor are fixed to the BEDT-TTF molecules, we can determine the principal values of the $g$ tensor for $\beta^{\prime \prime}$-Ga salt as $g=(2.016,2.010,2.001)$. Good agreement with a previous study [21] suggests that all EPR spectra can be explained by the anisotropic $g$ tensor and the double-layer crystal structure of $\beta^{\prime \prime}$-Ga salt, meaning that our crystal is single phase and the electronic state is uniform.

Next, we measured the temperature dependence of the EPR spectrum in the field applied to the direction $45^{\circ}$ rotated from the $b$ axis ( $45^{\circ}$ field). In this field direction, $T_{c}$ is suppressed below $3 \mathrm{~K}$ by a field of approximately $300 \mathrm{mT}$. With increasing temperature, the peak separation becomes small, as shown in Fig. 2, and a single peak was observed at temperatures higher than $8.5 \mathrm{~K}$. We found a trace of the two-peak structure at $8 \mathrm{~K}$ as the wiggle around the center of the spectrum at $344.4 \mathrm{mT}$. Therefore, the peak positions were determined by the two-peak Lorentzian fit for the spectra below $8 \mathrm{~K}$ [solid symbols in Fig. 3(a)], and by the single-peak Lorentzian fit above $8.5 \mathrm{~K}$ [open symbols in Fig. 3(a)]. The abrupt increase in the peak separation below $8 \mathrm{~K}$ clearly 


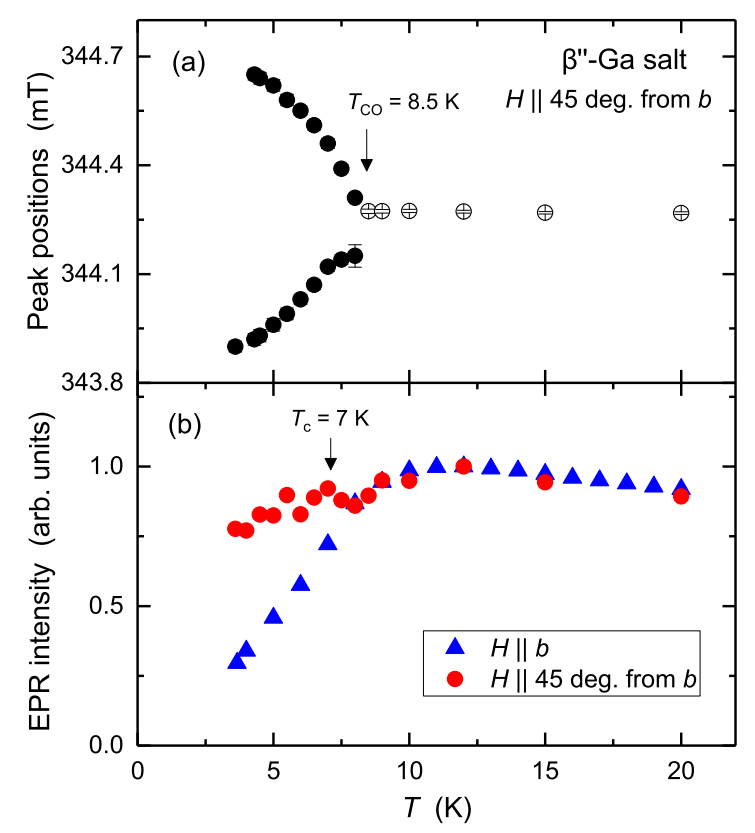

FIG. 3. (a) The temperature dependence of the EPR peak fields. Abrupt peak splitting was observed below $T_{\mathrm{CO}}=8.5 \mathrm{~K}$. (b) The temperature dependence of the EPR intensity in the $b$-axis field, and $45^{\circ}$ field. The intensity is normalized at $12 \mathrm{~K}$ to correct the intensity difference due to the sample shape. The SC transition is detected as the reduction of EPR intensity below $T_{c}$ in the $b$-axis field.

evidences a phase transition. This anomaly agrees with the charge ordering transition at $T_{\mathrm{CO}}=8.5 \mathrm{~K}$ previously observed from the ${ }^{13} \mathrm{C}$ NMR study [18]. The origin of the EPR peak splitting will be discussed later.

As the charge ordering anomaly is successfully observed in the EPR spectrum, then we compare the EPR spectra in the $b$-axis and $45^{\circ}$ fields to unveil the relationship between the CO and SC states. Typical EPR spectra for $b$-axis fields are presented in the Supplemental Material [20]. In the $b$-axis field of $345 \mathrm{mT}, T_{c}$ does not change because of the extremely high upper critical field $\left(B_{c 2}>30 \mathrm{~T}\right)$. The effect of the SC transition was observed in the EPR spectrum as a reduction of the integrated intensity below 7 K [Fig. 3(b)]. Such a decrease in intensity was not observed in the $45^{\circ}$ field, because $T_{c}$ is suppressed below $3 \mathrm{~K}$. This result confirms that the electronic spins that would show superconductivity in zero field contribute to the EPR intensity when superconductivity is suppressed by the $45^{\circ}$ field. Thus, if the SC part of the sample did not show the charge ordering transition, which is the case for the macroscopic phase segregation, an additional EPR peak originating from the electrons in a normal metallic state should be observed at the center of the two-peak spectrum. However, such an extra contribution was not observed at the lowest temperature of $3.6 \mathrm{~K}$, as shown at the bottom of Fig. 2. The clear two-peak spectrum in the $45^{\circ}$ field allows us to conclude that the SC state coexists uniformly with the CO state. We note that the EPR intensity decreases gradually below $T_{c}$ in the $b$-axis field, and finite intensity remains even at $3.6 \mathrm{~K}$. This behavior is contrasting with the conventional behavior expected for a homogeneous SC state, in which EPR signal should disappear. The EPR in the SC state may originate from the nearly localized electrons in the CO state, for instance, the pin site in the pinball liquid state. We exclude the possibility that all EPR spectra originates from the free spins induced by impurity or defects, because the EPR intensity increases from $20 \mathrm{~K}$ up to room temperature, which is in contrast to the Curie behavior expected for the free spins [20]. In fact, a typical Curie-Weiss-type temperature dependence was observed above $T_{c}$ in the C- and Al-doped $\mathrm{MgB}_{2}$ [22-24], in which the EPR signal originates from the defects and/or impurities.

Now, to study in detail the origin of the EPR peak splitting in the $\mathrm{CO}$ state, we fit the EPR spectra using the Bloch model with cross spin relaxation between the neighboring layers A and B [Fig. 1(c)] [25]. The time evolutions of the electron magnetization on the weakly coupled layers A and $\mathrm{B}, \boldsymbol{M}^{\mathrm{A}}, \boldsymbol{M}^{\mathrm{B}}$, are written as

$$
\begin{gathered}
\frac{d \boldsymbol{M}^{\mathrm{A}}}{d t}=\frac{g^{\mathrm{A}} \mu_{B}}{h}\left(\boldsymbol{M}^{\mathrm{A}} \times \boldsymbol{B}\right)-\boldsymbol{R}^{\mathrm{A}}+\frac{\Delta \boldsymbol{M}^{\mathrm{AB}}}{T_{\mathrm{X}}}, \\
\frac{d \boldsymbol{M}^{\mathrm{B}}}{d t}=\frac{g^{\mathrm{B}} \mu_{B}}{h}\left(\boldsymbol{M}^{\mathrm{B}} \times \boldsymbol{B}\right)-\boldsymbol{R}^{\mathrm{B}}+\frac{\Delta \boldsymbol{M}^{\mathrm{BA}}}{T_{\mathrm{X}}},
\end{gathered}
$$

where $\boldsymbol{R}^{\alpha}=\left[M_{x}^{\alpha} / T_{2}, M_{y}^{\alpha} / T_{2},\left(M_{z}^{\alpha}-M_{0}\right) / T_{1}\right]$ with $\alpha=\mathrm{A}$, B layers, $\Delta \boldsymbol{M}^{\mathrm{AB}}=\boldsymbol{M}^{\mathrm{B}}-\boldsymbol{M}^{\mathrm{A}}$, and $g^{\alpha}, \mu_{B}$, and $h$ are the $g$ factor for the $\alpha$ layer, the Bohr magneton, and the Planck's constant. When the intrinsic spin relaxation time $T_{2}$ becomes short, the whole spectrum is broadened in proportion to $1 / T_{2}$, and the broad spectrum smears out the two-peak structure. Contrastingly, when the cross relaxation time $T_{\mathrm{X}}$ becomes faster than the timescale equivalent to the spectrum separation, the twopeak structure is lost due to the dynamical narrowing effect, and a single sharp peak will be observed. The sharp EPR spectra at high temperatures above $8.5 \mathrm{~K}$ in the present study are explained by this dynamical narrowing effect. Figure 4(a) shows the temperature dependence of $T_{\mathrm{X}}$ determined by fitting the experimental spectra with the coupled Bloch model [20]. The gray region in Fig. 4(a) represents the timescale shorter than the spectrum separation of approximately $20 \mathrm{MHz}$.

In the $\mathrm{CO}$ state, the temperature dependence of $1 / T_{\mathrm{X}}$ shows an exponential behavior with a gap energy of $\Delta / k_{B}=16 \mathrm{~K}$. This gap size is consistent with that expected for the weakcoupling charge density wave state, $2 \Delta / k_{B} T_{\mathrm{CO}}=3.8$. The charge gap was detected in the interplane spin exchange channel because the already small interplane transfer integral in the metallic state is completely lost in the $\mathrm{CO}$ state due to the partial charge localization, whereas for the in-plane spin relaxation $T_{2}$ we found that the spectrum width is invariant above and below $T_{\mathrm{CO}}$, which leads us to conclude that $T_{2}$ is not significantly modified by a charge ordering transition. The invariant $T_{2}$ allows us to exclude the possibility of a magnetic phase transition. This anisotropic behavior is consistently explained by the threefold $\mathrm{CO}$ state, in which partial carrier localization immediately extinguishes the weak interplane transfer channel, while maintaining the coherent in-plane transfer channel.

The anisotropy developing in the $\mathrm{CO}$ state is also observed from the electrical transport experiment, as shown in Fig. 4(b) [20]. The in-plane resistance $\left(R_{\text {perp }}\right)$ and interplane resistance $\left(R_{\text {para }}\right)$ were measured in magnetic fields of 0.3 and $2.5 \mathrm{~T}$ applied perpendicular to the conducting plane to suppress $T_{c}$. 


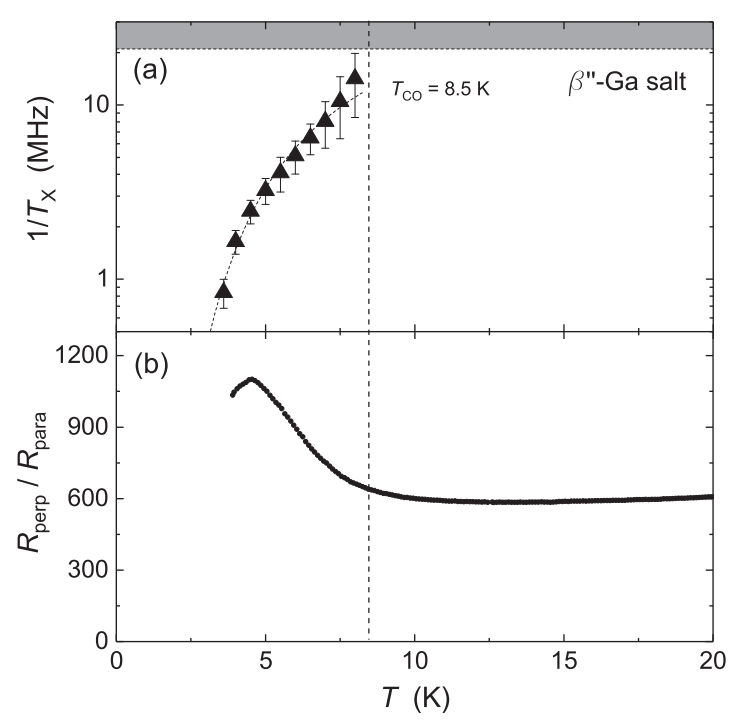

FIG. 4. The temperature dependence of (a) the cross relaxation rate $1 / T_{\mathrm{X}}$, and (b) the ratio between the in-plane and interplane resistance. The gray area in (a) represents the frequency that corresponds to the EPR peak separation at the lowest temperature, above which $1 / T_{\mathrm{X}}$ cannot be determined because of the motional narrowing. At low temperatures, $1 / T_{\mathrm{X}}$ follows an exponential function with a gap energy of $16 \mathrm{~K}$ as shown by the dotted line.

The increase in the ratio $R_{\text {perp }} / R_{\text {para }}$ below $T_{\mathrm{CO}}$ means that the interplane resistivity increases due to the gap opening in the interplane transfer channel. A kink was observed at a lower temperature of $4.5 \mathrm{~K}$ because of the precursor to the SC transition. The anomaly at $T_{\mathrm{CO}}$ was not clearly observed in the previously reported in-plane resistivity measurements $[15,16]$, because the highly conducting in-plane channel is not significantly modified in the $\mathrm{CO}$ state, which is consistent with the invariant $T_{2}$. The highly conducting in-plane channel is also suggested from the quantum oscillation study, in which a clear Shubnikov-de Haas oscillation was observed even in the CO state, where resistivity shows a semiconducting temperature dependence $[19,26]$. Observation of the Fulde-Ferrell-LarkinOvchinnikov superconducting state in high magnetic fields is more evidence of a clean electronic state in the conducting plane [27].

Finally, a question is whether the $\mathrm{CO}$ state supports or suppress superconductivity. In a series of $\beta^{\prime \prime}$-type BEDT-TTF salts with various guest molecules and metallic ions, a $T_{c}$ higher than $5 \mathrm{~K}$ is found only in the salts with a resistivity upturn at low temperatures [26], whereas in the $\beta^{\prime \prime}$-Rh salt with $T_{c}=2.5 \mathrm{~K}$, almost no resistivity upturn was observed [28]. As the charge ordering transition increases the SC transition temperatures, we suggest the importance of the exotic electronic state with a spatial charge modulation $[12,13]$ to induce this unconventional type of superconductivity.

To conclude, we performed an EPR experiment for the organic superconductor $\beta^{\prime \prime}$-Ga salt, and clearly detected a charge ordering transition at $T_{\mathrm{CO}}=8.5 \mathrm{~K}$. In the $\mathrm{CO}$ state, the interplane spin exchange channel is gapped as a result of the partial charge localization. In the same sample, we observed the SC transition as the reduction of EPR intensity below $T_{c}$. We found only a single spectrum component both in the SC and $\mathrm{CO}$ states, which suggests a uniform coexistence between superconductivity and charge order. To understand this exotic coexisting state, we suggest a threefold CO state, in which partial charge localization coexists with high conductivity.

We would like to acknowledge Y. Oshima, and L. Martin for fruitful discussions, and M. Fujiwara for the support in carrying out the EPR experiment at the Institute for Molecular Science. This study was partly supported by the Suhara Memorial Foundation.
[1] H. Kobayashi, R. Kato, A. Kobayashi, Y. Nishio, K. Kajita, and W. Sasaki, Chem. Lett. 15, 789 (1986).

[2] M. Kurmoo, M. J. Rosseinsky, P. Day, P. Auban, W. Kang, D. Jérôme, and P. Batail, Synth. Met. 27, A425 (1988).

[3] H. Mori, S. Tanaka, and T. Mori, Phys. Rev. B 57, 12023 (1998).

[4] N. D. Mathur, F. M. Groshe, S. R. Julian, I. R. Walker, D. M. Freye, R. K. Haselwimmer, and G. G. Lonzarich, Nature (London) 394, 39 (1998).

[5] J. Merino and R. H. McKenzie, Phys. Rev. Lett. 87, 237002 (2001).

[6] S. Kaiser, M. Dressel, Y. Sun, A. Greco, J. A. Schlueter, G. L. Gard, and N. Drichko, Phys. Rev. Lett. 105, 206402 (2010).

[7] T. Kakiuchi, Y. Wakabayashi, H. Sawa, T. Takahashi, and T. Nakamura, J. Phys. Soc. Jpn. 76, 113702 (2007).

[8] Y. Nogami, J.-P. Pouget, M. Watanaba, K. Oshima, H. Mori, S. Tanaka, and T. Mori, Synth. Met. 103, 1911 (1999).

[9] K. Miyagawa, A. Kawamoto, and K. Kanoda, Phys. Rev. B 62 , R7679 (2000).

[10] T. Mori, J. Phys. Soc. Jpn. 72, 1469 (2003).

[11] M. Kaneko and M. Ogata, J. Phys. Soc. Jpn. 75, 014710 (2006).

[12] C. Hotta, N. Furukawa, A. Nakagawa, and K. Kubo, J. Phys. Soc. Jpn. 75, 123704 (2006).
[13] J. Merino, A. Greco, N. Drichko, and M. Dressel, Phys. Rev. Lett. 96, 216402 (2006).

[14] M. Watanabe, Y. Noda, Y. Nogami, and H. Mori, J. Phys. Soc. Jpn. 73, 116 (2004).

[15] H. Akutsu, A. Akutsu-Sato, S. S. Turner, D. Le Pevelen, P. Day, V. Laukhin, A.-K. Klehe, J. Singleton, D. A. Tocher, M. R. Probert, and J. A. K. Howard, J. Am. Chem. Soc. 124, 12430 (2002).

[16] Y. Ihara, H. Seki, and A. Kawamoto, J. Phys. Soc. Jpn. 82, 083701 (2013).

[17] Y. Ihara, Y. Futami, and A. Kawamoto, J. Phys. Soc. Jpn. 85, 014601 (2016).

[18] Y. Ihara, M. Jeong, H. Mayaffre, C. Berthier, M. Horvatić, H. Seki, and A. Kawamoto, Phys. Rev. B 90, 121106(R) (2014).

[19] A. I. Coldea, A. F. Bangura, J. Singleton, A. Ardavan, A. Akutsu-Sato, H. Akutsu, S. S. Turner, and P. Day, Phys. Rev. B 69, 085112 (2004)

[20] See Supplemental Material at http://link.aps.org/supplemental/ 10.1103/PhysRevB.100.060505 for technical details of resistivity measurement and EPR spectrum analyses, which includes Refs. [29,30].

[21] N. Kinoshita, M. Tokumoto, H. Anzai, and G. Saito, J. Phys. Soc. Jpn. 54, 4498 (1985). 
[22] A. Bateni, S. Repp, R. Thomann, S. Acar, E. Erdem, and M. Somer, Appl. Phys. Lett. 105, 202605 (2014).

[23] A. Bateni, E. Erdem, S. Repp, S. Acar, I. Kokal, W. Häßler, S. Weber, and M. Somer, J. Appl. Phys. 117, 153905 (2015).

[24] A. Bateni, E. Erdem, S. Repp, S. Weber, and M. Somer, Appl. Phys. Lett. 108, 202601 (2016).

[25] Á. Antal, T. Fehér, E. Tátrai-Szekeres, F. Fülöp, B. Náfrádi, L. Forró, and A. Jánossy, Phys. Rev. B 84, 075124 (2011).

[26] A. F. Bangura, A. I. Coldea, J. Singleton, A. Ardavan, A. Akutsu-Sato, H. Akutsu, S. S. Turner, P. Day, T. Yamamoto, and K. Yakushi, Phys. Rev. B 72, 014543 (2005).
[27] S. Uji, Y. Iida, S. Sugiura, T. Isono, K. Sugii, N. Kikugawa, T. Terashima, S. Yasuzuka, H. Akutsu, Y. Nakazawa, D. Graf, and P. Day, Phys. Rev. B 97, 144505 (2018).

[28] L. Martin, A. L. Morritt, J. R. Lopez, H. Akutsu, Y. Nakazawa, S. Imajo, and Y. Ihara, Inorg. Chem. 56, 717 (2017).

[29] Y. Goto, A. Miura, C. Moriyoshi, Y. Kuroiwa, T. D. Matsuda, Y. Aoki, and Y. Mizuguchi, Sci. Rep. 8, 12852 (2018).

[30] A. J. S. Machado, S. T. Renosto, C. A. M. dos Santos, L. M. S. Alvas, and Z. Fisk, in Superconductors - Materials, Properties and Applications, edited by A. Gabovich (IntechOpen, London, 2012), Chap. 3. 